\title{
Dificuldades para alimentação saudável entre usuários da atenção básica em saúde e fatores associados
}

\author{
Difficulties in ensuring a healthy diet \\ among primary health care users and associated factors
}

Ivana Loraine Lindemann ${ }^{1}$

Riceli Rodeghiero Oliveira ${ }^{2}$

Raúl Andres Mendoza-Sassi ${ }^{3}$

${ }^{1}$ Programa de PósGraduação em Ciências da Saúde, Faculdade de Medicina, Universidade Federal do Rio Grande (FURG). R. José Reolon 921/407, Centro. 99010-464, Passo Fundo RS Brasil. ivanaloraine@hotmail.com ${ }^{2}$ Curso de Nutrição,

Faculdade de Nutrição, Universidade Federal de Pelotas. Pelotas RS Brasil. ${ }^{3}$ Faculdade de Medicina, FURG. Rio Grande RS Brasil.
Abstract This is a straightforward cross-sectional study of health services to assess the prevalence of perception of difficulties in ensuring a healthy diet, associated factors and respective motives among a sample of 1,246 adult and elderly primary care users. The prevalence of perception of difficulties was 31.1\% (95\% CI 28.5-33.6) and associated factors were female gender $(P R=1.69$; 95\% CI 1.28-2.25), adulthood ( $P R=1.33$; 95\% CI 1.11-1.59), income, with the most marked effect in the highest quintile $(P R=0.63 ; 95 \%$ CI 0.47-0.83), a higher number of self-reported chronic diseases $(P R=1.31 ; 95 \%$ CI 1.03-1.67), food insecurity $(P R=2.28 ; 95 \%$ CI 1.93-2.69), not receiving food guidance $(P R=0.75 ; 95 \% C I$ 0.63 to 0.89 ) and not reading food labels ( $P R=$ 1.42; 95\% CI 1.08-1.86). Among those who had difficulties, the reasons mentioned were high cost of foods considered to be healthy (57.6\%), insufficient willpower (49.4\%), need to give up food considered unhealthy (41.6\%), lack of time (35.7\%) and insufficient knowledge (31.3\%). The results show the importance of the awareness of health professionals about the difficulties of users and the need for adequate actions to promote a healthy diet in order to avoid standard patient care procedures.

Key words Primary health care, Health promotion, Nutrition in public health, Eating behavior
Resumo Estudo transversal de base de serviços de saúde sobre a prevalência de percepção dificuldades para ter uma alimentação saudável, seus fatores associados e seus motivos, em uma amostra de 1.246 adultos e idosos usuários da atenção básica. A prevalência de percepção dificuldades foi de 31,1\% (IC95 28,5-33,6) e os fatores associados foram sexo feminino ( $R P=1,69$; IC95 1,28-2,25), idade adulta $(R P=1,33$; IC95 1,11-1,59), renda, sendo o maior efeito no quintil mais elevado $(R P=0,63$; IC95 0,47-0,83), maior número de doenças crônicas autorreferidas $(R P=1,31$; IC95 1,03-1,67), insegurança alimentar $(R P=2,28$; IC95 1,93-2,69), não recebimento de orientação alimentar $(R P=0,75 ; 0,63-0,89)$ e hábito de não ler rótulos de alimentos $(R P=1,42 ; 1,08-1,86)$. Dentre os que tinham dificuldades, os motivos mencionados foram custo elevado dos alimentos considerados saudáveis (57,6\%), força de vontade insuficiente (49,4\%), necessidade de abrir mão de alimentos considerados não saudáveis (41,6\%), falta de tempo (35,7\%) e conhecimento insuficiente (31,3\%). Os resultados evidenciam a importância do conhecimento dos profissionais de saúde acerca das dificuldades dos usuários e da necessidade de adequação das ações de promoção da alimentação saudável, a fim de evitar condutas padronizadas.

Palavras-chave Atenção primária à saúde, Promoção da saúde, Nutrição em saúde pública, Comportamento alimentar 


\section{Introdução}

Evidências científicas têm demonstrado a ascensão das doenças crônicas não transmissíveis $(\mathrm{DCNT})^{1,2}$, em decorrência de um conjunto de fatores de risco, dentre os quais a alimentação $0^{3,4}$, que muitas vezes é também parte do tratamento não farmacológico de várias dessas enfermidades, salientando assim a sua importância ${ }^{5,6}$. Além disso, tem se observado significativas modificações no padrão alimentar da população em muitos países ${ }^{1,7}$, inclusive no Brasil, onde se destaca o consumo excessivo de açúcares, gorduras, produtos industrializados e preparações ricas em sódio, a redução da ingestão do feijão com arroz e a insuficiência de frutas e hortaliças ${ }^{8-10}$.

Nesse contexto, a Organização Mundial da Saúde (OMS) propôs a Estratégia Global para Alimentação, Atividade Física e Saúde, atribuindo aos governos de cada país a responsabilidade de elaborar guias para promoção da alimentação saudável e prática de atividade física ${ }^{1}$. Assim, o Ministério da Saúde (MS) do Brasil publicou o Guia Alimentar para a População Brasileira, o qual contém as diretrizes alimentares oficiais para os brasileiros ${ }^{11}$.

Sabe-se que as recomendações em termos do que se configura uma alimentação saudável, oficiais ou não, por razões ligadas à saúde ou não, são divulgadas à população de forma ampla e diversa, incluindo, políticas, programas e campanhas do MS, profissionais de saúde e mídia' ${ }^{12,13}$, o que leva a crer que, de uma forma ou de outra, a maior parte das pessoas tem acesso a esse conhecimento. No entanto, conforme mencionado anteriormente, as pesquisas continuam demonstrando que a alimentação habitual do brasileiro segue um padrão considerado não saudável.

Tais constatações reafirmam que, embora o conhecimento favoreça o desenvolvimento e a manutenção de novas atitudes alimentares, sua influência não é incondicional ${ }^{14} \mathrm{e}$, muitas vezes, mesmo tendo recebido a orientação de um profissional de saúde, a adesão às recomendações enfrenta dificuldades, pois a efetiva mudança de comportamento caracteriza-se como um processo complexo ${ }^{15}$. No Brasil, dentre usuários da atenção básica de um município de grande porte, 20,3\% receberam orientação para alimentação saudável, dos quais aproximadamente $10 \%$ seguiam todas as recomendações, enquanto que a metade não seguia nenhuma. As principais dificuldades mencionadas foram a falta de tempo e de recursos financeiros e a dificuldade de mudar hábitos e de controlar impulsos alimentares².
Estudo realizado na Espanha apontou prevalência de dificuldades para alimentação saudável em torno de $80 \%$. As principais barreiras citadas foram horário irregular de trabalho, falta de força de vontade, custo dos produtos e necessidade de abrir mão de alimentos de que gosta ${ }^{16}$. Entre a população Romena, os principais motivos foram ligados ao custo e à necessidade de abrir mão de determinados alimentos ${ }^{17}$.

Dificuldades com alimentação saudável também foram observadas na Ásia Ocidental, repetindo-se fatores como falta de vontade, de tempo e de recursos financeiros, o que indica que $o$ problema é universal, transcendendo aspectos culturais ${ }^{18,19}$.

Apesar da relevância do tema, conforme observado na literatura disponível, poucos foram os estudos que investigaram a prevalência e o tipo de dificuldades para alimentação saudável, bem como seus fatores associados, especialmente entre brasileiros e usuários dos sistemas públicos de saúde.

Considerando o contexto da atenção básica em saúde no Brasil, em que as políticas públicas têm enfatizado a promoção da alimentação saudável e reafirmado a responsabilidade dos profissionais, especialmente os vinculados ao Sistema Único de Saúde (SUS), mais especificamente os que atuam na atenção básica ${ }^{3,10,20,21}$, é importante que, além de orientar, se conheçam as dificuldades dos usuários para terem uma alimentação saudável, para que de posse dessas informações seja possível adequar as estratégias de intervenção. Assim, o objetivo do presente estudo foi determinar a prevalência de percepção de dificuldades para ter uma alimentação saudável, seus fatores associados e seus motivos, entre usuários da atenção básica de saúde.

\section{Metodologia}

Realizamos um estudo transversal de base de serviços de saúde, de maio a outubro de 2013, na cidade de Pelotas, estado do Rio Grande do Sul, Brasil. Situada ao Sul do estado, de acordo com o censo demográfico de 2010, Pelotas tinha uma população estimada em 328.275 habitantes e Índice de Desenvolvimento Humano de $0,739^{22}$. No ano de 2013 a rede contava com 36 Unidades Básicas de Saúde (UBS) na zona urbana, sendo que 14 destas tinham equipes da Estratégia de Saúde da Família (ESF), indicando assim uma cobertura de $38,9 \%$. 


\section{População de estudo, cálculo de amostra e amostragem}

Consideramos como elegíveis os usuários de todas as UBS da zona urbana, de ambos os sexos, com idade igual ou superior a 20 anos. Excluímos mulheres em período de gestação ou de lactação e portadores de deficiência física ou mental, devido à dieta e avaliação nutricional diferenciada ou dificuldade em responder o questionário.

O tamanho amostral foi calculado com o Programa Epi Info 6.04 (Centers for Disease Control and Prevention, Atlanta, Estados Unidos), levando-se em consideração diferentes fatores de risco, risco relativo de 2,0, nível de confiança de $95 \%$, poder de $80 \%$, relação de não expostos para expostos de até 1:9 e prevalência esperada do desfecho em não expostos de no mínimo 13\%. A amostra necessária seria de 936, o que acrescido de $10 \%$ para perdas e $25 \%$ para fatores de confusão, totalizou 1.264 participantes. Todas as UBS da zona urbana foram incluídas e a amostragem foi realizada em duas etapas. Primeiro foi utilizada uma amostragem do tipo aleatória proporcional, tomando-se como critério para definir o número de usuários a ser entrevistado em cada UBS o número médio de procedimentos realizado no mês anterior ao início da coleta de dados. Numa segunda etapa foi utilizada uma amostragem de conveniência, sendo que as duplas de entrevistadores se deslocavam até cada uma das UBS e entrevistavam de forma consecutiva os usuários presentes para consulta nesse dia. Não se atingindo o n definido para essa UBS, os entrevistadores retornavam no dia seguinte, e assim consecutivamente até completar o n estipulado, sem repetição de usuários.

\section{Variáveis estudadas}

Como desfecho do estudo foi considerada a percepção por parte do usuário de dificuldades para ter uma alimentação saudável. Para isso foi utilizada a pergunta: $O(a) \operatorname{Sr}(a)$ tem dificuldades para ter uma alimentação saudável? Em caso de resposta afirmativa, foi perguntado quais eram as dificuldades, tendo como opções de resposta conhecimento insuficiente, falta de tempo, força de vontade insuficiente, custo elevado dos alimentos considerados saudáveis e necessidade de abrir mão de alimentos considerados não saudáveis, as quais eram lidas aos respondentes.

Analisamos a associação do desfecho com variáveis sociodemográficas, de situação de saúde do usuário, de conhecimento sobre saúde e tipo de serviço utilizado e, de hábitos de vida. As variáveis sociodemográficas contemplaram sexo, idade (medida em anos completos e categorizada em 20-59, 60 anos ou mais), cor da pele autorreferida (branca, preta e outras), número de pessoas no domicílio (1-3, 4 ou mais), situação conjugal (com cônjuge, sem cônjuge), quintis de renda familiar mensal per capita (sendo o $1^{\circ}$ o menos favorecido economicamente), escolaridade (ensino fundamental, médio, superior ou mais) e ocupação (trabalha, não trabalha).

$\mathrm{Na}$ situação de saúde do usuário analisamos número de DCNT autorreferidas e diagnosticadas pelo médico (obesidade, diabetes, hipertensão, hipercolesterolemia, hipertrigliceridemia e doença cardíaca, categorizada em nenhuma, 1-2 e 3 ou mais), estado nutricional (avaliado pelo peso e altura autorreferidos, classificado pelo Índice de Massa Corporal $^{20}$ e categorizado em eutrofia ou excesso de peso) e insegurança alimentar ( sim ou não, avaliada a partir da escala proposta por Bickel et al. ${ }^{23}$ ).

Em relação ao conhecimento sobre saúde e tipo de serviço utilizado, foi considerado se o usuário ouviu falar nos Dez Passos da Alimentação Saudável ${ }^{24}$ (sim ou não), se recebe informações sobre saúde ( mações sobre nutrição ( $\operatorname{sim}$ ou não), se recebe orientação para alimentação saudável (sim ou não) e tipo de modelo de atenção à saúde (tradicional ou saúde da família, segundo a UBS em que é atendido).

Como hábitos de vida foram incluídos leitura de rótulos de alimentos (nunca, às vezes, sempre), hábito de fumar ( $\operatorname{sim}$ ou não, sem considerar frequência e quantidade), consumo de bebidas alcoólicas (sim ou não, sem considerar frequência e quantidade) e prática de atividade física (sim ou não, sem considerar atividade e frequência).

\section{Instrumento e procedimentos}

Os dados foram coletados por meio da aplicação de um questionário pré-codificado, testado com usuários de um ambulatório de saúde da cidade. As seis duplas de entrevistadores, devidamente treinadas, após apresentarem-se à recepção de cada UBS, identificavam os sujeitos elegíveis e os convidavam a participar, mediante consentimento livre e esclarecido. Em caso de recusa, duas novas tentativas eram feitas, pelo outro entrevistador da dupla e pelo supervisor de campo. Não houve reposição de perdas. As entrevistas foram feitas de segunda a sexta-feira, nos turnos manhã e tarde, na sala de espera, antes das 
consultas, e, $10 \%$ das entrevistas foram refeitas para o controle de qualidade.

\section{Processamento e análise estatística dos dados}

Os dados foram duplamente digitados em banco criado no Programa EpiData 3.1 (EpiData Association, Dinamarca) e a análise estatística foi realizada com o Stata, versão 11 (Stata Corp., College Station, Estados Unidos). Inicialmente foi realizada a descrição da amostra e calculada a prevalência do desfecho e seu intervalo de confiança de 95\% (IC95). Na análise bivariada foram calculadas as Razões de Prevalências (RP) brutas e seus IC95. Para proceder o ajuste dos fatores de confusão, foi utilizada a Regressão de Poisson com variância robusta, considerando a opção para amostra por conglomerados do Stata (robust). A análise que gerou as RP ajustadas e seus IC95, foi do tipo backward stepwise, seguindo um modelo hierárquico ${ }^{25}$ pré-estabelecido, composto de quatro níveis de determinação. No primeiro nível incluímos todas as variáveis sociodemográficas, no segundo, as referentes à situação de saúde, no terceiro, adicionamos as variáveis de conhecimento sobre saúde e tipo de serviço utilizado e, no nível mais proximal, as relacionadas aos hábitos de vida. Aquelas que apresentassem um $\mathrm{p} \leq 0,20$ foram mantidas para o ajuste com o nível seguinte com a finalidade de evitar a confusão negativa. No caso de variáveis categóricas politômicas, quando houve ordenamento entre as categorias foi realizado o teste de Wald para tendência linear e, quando não houve, foi aplicado o teste de heterogeneidade. Em todos os testes de significância foi considerado um valor de $\mathrm{p}<$ 0,05 de um teste bicaudal.

O protocolo de estudo foi aprovado pelo Comitê de Ética em Pesquisa na Área da Saúde, da Universidade Federal do Rio Grande.

\section{Resultados}

Dentre os 1.264 usuários da atenção básica selecionados para participar, 1.246 aceitaram responder ao questionário (1,4\% de perdas). Conforme se observa na Tabela 1 , predominou na amostra o sexo feminino $(83,7 \%)$, o grupo de idade mais jovem $(77,8 \%)$, a cor da pele branca $(63,3 \%)$ e a presença de cônjuge $(60,2 \%)$. A renda per capita variou de R\$ 0,0 a R \$3.333,3 (Média 496,9 \pm DP $344,1)$, a maior parte tinha ensino fundamental $(66,8 \%)$ e não estava trabalhando $(68,2 \%)$.
O diagnóstico médico de alguma DCNT foi referido pela maior parte dos entrevistados $(53,8 \%), 61,0 \%$ tinham excesso de peso e $86,0 \%$ apresentaram segurança alimentar. A maioria nunca ouviu falar nos Dez Passos da Alimentação Saudável (87,6\%), 62,6\% recebiam informações sobre saúde, mas $62,4 \%$ responderam não receber informações sobre nutrição. O recebimento de orientação para práticas alimentares saudáveis foi mencionado por $57,9 \%$ dos entrevistados e 53,7\% eram atendidos pela ESF. Às vezes liam rótulos de alimentos $41,6 \%$ dos participantes, $23,4 \%$ eram fumantes, $14,9 \%$ consumiam bebida alcoólica e 25,5\% faziam atividade física (Tabela $1)$.

Do total de entrevistados, 31,1\% (IC95 28,5$33,6)$ percebiam dificuldades para ter uma alimentação saudável (Tabela 1). Dentre os motivos pelos quais referiram dificuldades, 57,6\% (IC95 52,7-62,6) mencionaram o custo elevado dos alimentos considerados saudáveis, 49,4\% (IC95 44,4-54,4) a força de vontade insuficiente, 41,6\% (IC95 36,7-46,5) a necessidade de abrir mão de alimentos considerados não saudáveis, 35,7\% (IC95 31,7-40,5) a falta de tempo e 31,3\% (IC95 26,6-35,9) o conhecimento insuficiente.

$\mathrm{Na}$ análise da associação dos fatores de risco com o desfecho observamos que após o ajuste para fatores de confusão algumas mudanças ocorreram em relação à análise bruta. As variáveis do primeiro nível que permaneceram no modelo final foram o sexo feminino, com $69,0 \%$ maior probabilidade de percepção de dificuldade ( $\mathrm{RP}=$ 1,69; IC95 1,28-2,25) e a idade adulta com 33,0\% $(\mathrm{RP}=1,33$; IC95 1,11-1,59). No mesmo nível, a probabilidade de percepção de dificuldades para alimentação saudável variou de forma significativa entre os estratos de renda ( $\mathrm{p}$ de heterogeneidade 0,009). A probabilidade do desfecho entre quintis de renda foi reduzida em $20,0 \%$ no $2^{\circ}$ quintil ( $\mathrm{RP}=0,80$; IC95 0,66-0,97), em 27,0\% no $3^{\circ}\left(\mathrm{RP}=0,73\right.$; IC95 0,56-0,96), em $24,0 \%$ no $4^{\circ}$ $(\mathrm{RP}=0,76$; IC95 0,53-1,09) e em 37,0\% no mais elevado ( $\mathrm{RP}=0,63$; IC95 0,47-0,83) (Tabela 2).

No segundo nível permaneceu a variável portador de DCNT, sendo que aqueles com 3 ou mais doenças crônicas apresentaram 31,0\% maior probabilidade de perceberem dificuldades para alimentação saudável ( $\mathrm{RP}=1,31$; IC95 1,031,67). Também se manteve o efeito da insegurança alimentar, sendo a probabilidade do desfecho $128,0 \%$ maior entre os que apresentaram esta condição ( $\mathrm{RP}=2,28$; IC95 1,93-2,69) (Tabela 2).

No terceiro nível de análise, o modelo final incluiu os usuários que não receberam orienta- 
Tabela 1. Descrição de adultos e idosos usuários da rede urbana de Atenção Básica de Saúde. Pelotas, RS. 2014. $(\mathrm{n}=1.246)$.

\begin{tabular}{|c|c|c|c|}
\hline Variáveis & $\mathbf{n}$ & $\%$ & $\begin{array}{c}\text { Prevalência de dificuldades } \\
\text { para ter uma alimentação saudável (\%) }\end{array}$ \\
\hline & & & $31,1($ IC95 28,5-33,6) \\
\hline \multicolumn{4}{|l|}{ Demográficas } \\
\hline \multicolumn{4}{|l|}{ Sexo } \\
\hline Masculino & 203 & 16,3 & 17,7 \\
\hline Feminino & 1.043 & 83,7 & 33,7 \\
\hline \multicolumn{4}{|l|}{ Idade em anos completos } \\
\hline 60 ou mais & 277 & 22,2 & 22,4 \\
\hline $20-59$ & 969 & 77,8 & 33,5 \\
\hline \multicolumn{4}{|l|}{ Cor da pele autorreferida $(\mathrm{n}=1.244)^{*}$} \\
\hline Branca & 787 & 63,3 & 32,0 \\
\hline Preta e outras & 457 & 36,7 & 29,5 \\
\hline \multicolumn{4}{|l|}{ Número de pessoas no domicílio } \\
\hline $1-3$ & 692 & 55,5 & 29,6 \\
\hline 4 ou mais & 554 & 44,5 & 32,9 \\
\hline \multicolumn{4}{|l|}{ Situação conjugal } \\
\hline Sem cônjuge & 496 & 39,8 & 32,1 \\
\hline Com cônjuge & 750 & 60,2 & 30,4 \\
\hline \multicolumn{4}{|l|}{ Socioeconômicas } \\
\hline Quintis de renda familiar mensal per capita & Média & DP & \\
\hline 10 & 142,9 & 71,7 & 41,3 \\
\hline $2^{\circ}$ & 289,5 & 37,9 & 32,9 \\
\hline $3^{\circ}$ & 416,8 & 51,8 & 28,5 \\
\hline $4^{\circ}$ & 629,1 & 67,1 & 28,3 \\
\hline $5^{\circ}$ & $1.034,8$ & 304,8 & 23,2 \\
\hline \multicolumn{4}{|l|}{ Escolaridade } \\
\hline Ensino fundamental & 832 & 66,8 & 30,2 \\
\hline Ensino médio & 276 & 22,1 & 35,1 \\
\hline Ensino superior ou mais & 138 & 11,1 & 28,3 \\
\hline \multicolumn{4}{|l|}{ Ocupação } \\
\hline Não trabalha & 850 & 68,2 & 31,4 \\
\hline Trabalha & 396 & 31,8 & 30,3 \\
\hline \multicolumn{4}{|l|}{ Situação de saúde } \\
\hline \multicolumn{4}{|l|}{ Número de DCNT autorreferidas } \\
\hline Nenhuma & 576 & 46,2 & 29,5 \\
\hline $1-2$ & 486 & 39,0 & 30,9 \\
\hline 3 ou mais & 184 & 14,8 & 36,4 \\
\hline \multicolumn{4}{|l|}{ Estado nutricional $(\mathrm{n}=1.113)^{* *}$} \\
\hline Eutrofia & 434 & 39,0 & 30,4 \\
\hline Excesso de peso & 679 & 61,0 & 32,1 \\
\hline \multicolumn{4}{|l|}{ Insegurança alimentar $(\mathrm{n}=982)^{* * *}$} \\
\hline Não & 845 & 86,0 & 26,5 \\
\hline Sim & 137 & 14,0 & 65,0 \\
\hline
\end{tabular}

ção, com uma redução de $25,0 \%$ na probabilidade de perceberem dificuldades para alimentação saudável (RP = 0,75; IC95 0,63-0,89).

No quarto nível, a variável hábito de ler rótulos de alimentos manteve uma tendência linear de associação com o desfecho, sendo a probabili- dade de percepção de dificuldades para alimentação saudável $37,0 \%$ superior entre os que às vezes liam rótulos ( $\mathrm{RP}=1,37$; IC95 1,08-1,75) e 42,0\% entre os que nunca liam ( $\mathrm{RP}=1,42$; IC95 1,08 1,86). Com o ajuste, o hábito de fumar perdeu o efeito sobre o desfecho (Tabela 2). 


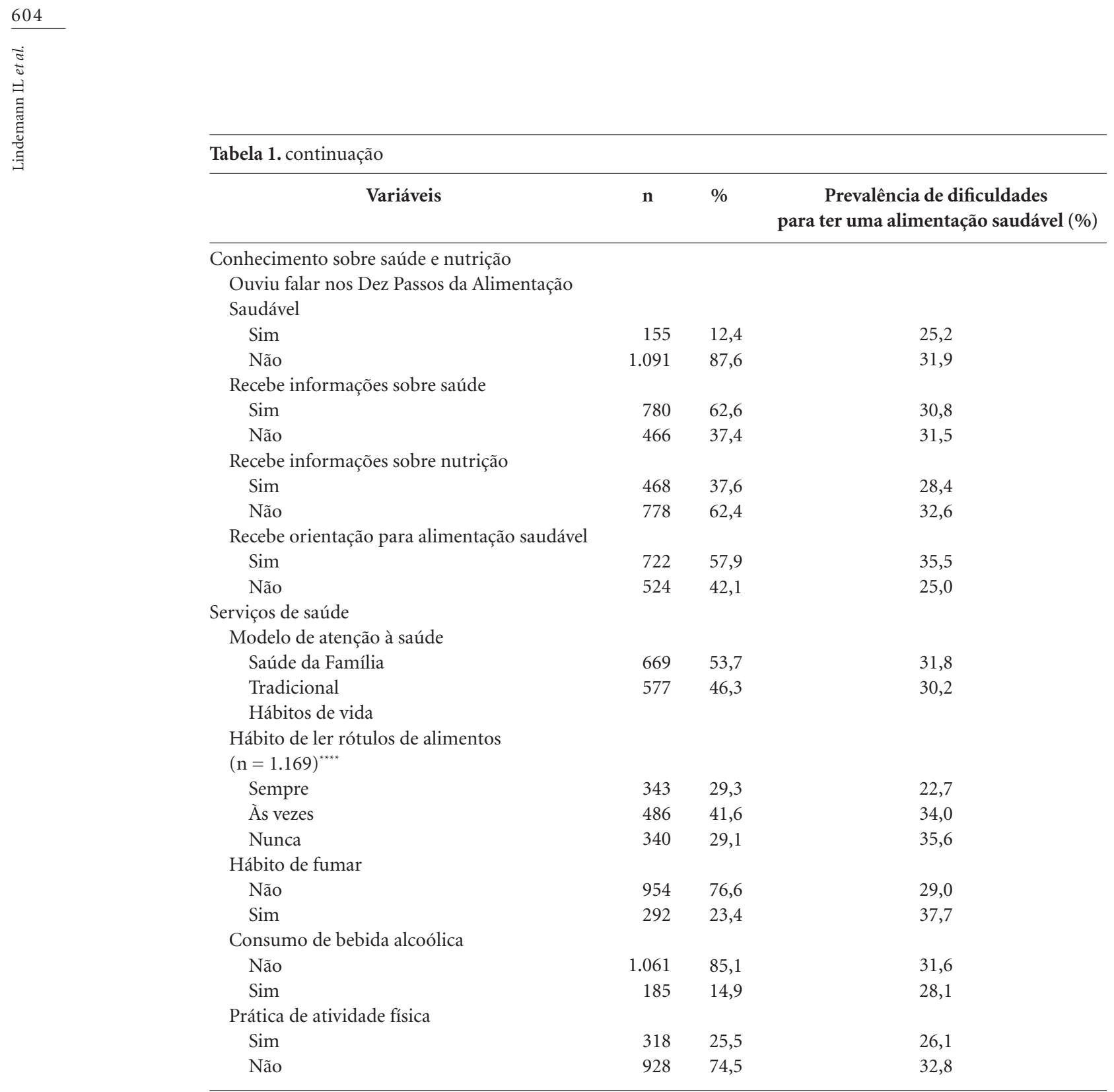

DCNT: doenças crônicas não transmissíveis; ${ }^{2}$ perdas; ${ }^{* *} 133$ perdas; ${ }^{* * *} 264$ perdas; ${ }^{* * *} 77$ perdas.

\section{Discussão}

Pesquisas de base de serviços de saúde que abordem a percepção de dificuldade para ter uma alimentação saudável na atenção básica no Brasil são escassas. Este estudo foi realizado em um município de porte médio na região Sul do Brasil e evidenciou que $1 / 3$ dos usuários da rede básica de saúde percebeu dificuldades para uma alimentação saudável, sendo que a metade ou mais assinalou como motivos para tal a força de vontade insuficiente e o elevado custo dos alimentos saudáveis, motivos relacionados a aspectos comportamentais e financeiros.
Mas antes de continuar discutindo nossos resultados, é necessário abordar as limitações metodológicas que podem tê-los influenciado. Ressaltamos em primeiro lugar a natureza transversal do estudo, de forma tal que os fatores identificados não podem ser considerados determinantes e sim apenas associados ao desfecho. Também devido ao tipo de delineamento, deve ser considerada a possibilidade de causalidade reversa entre o desfecho e alguns dos fatores estudados. Essas duas limitações metodológicas eram esperadas, e são inerentes à escolha do tipo de estudo mais utilizado para avaliar serviços de saúde. Outro aspecto a considerar é que, por ser 
Tabela 2. Análise bruta e ajustada de fatores associados a dificuldades para ter uma alimentação saudável, referidos por adultos e idosos usuários da rede urbana de Atenção Básica de Saúde. Pelotas, RS. 2014. (n = 1.246).

\begin{tabular}{|c|c|c|c|c|}
\hline Variáveis & RP (IC95) & $\mathbf{p}$ & RP (IC95) & $\mathbf{p}$ \\
\hline \multicolumn{5}{|l|}{$1^{\circ}$ nível $(\mathrm{n}=1.244)$} \\
\hline \multicolumn{5}{|l|}{ Demográficas $^{*}$} \\
\hline Sexo & & $<0,001^{\mathrm{a}}$ & & $<0,001^{\mathrm{a}}$ \\
\hline Feminino & $1,90(1,41-2,56)$ & & $1,69(1,28-2,25)$ & \\
\hline Idade em anos completos & & $<0,001^{\mathrm{a}}$ & & $0,002^{\mathrm{a}}$ \\
\hline $20-59$ & $1,50(1,27-1,77)$ & & $1,33(1,11-1,59)$ & \\
\hline Cor da pele autorreferida & & $0,263^{\mathrm{a}}$ & & $0,112^{\mathrm{a}}$ \\
\hline Preta e outras & $0,92(0,80-1,06)$ & & $0,89(0,77-1,03)$ & \\
\hline Número de pessoas no domicílio & & $0,221^{\mathrm{a}}$ & & $0,639^{\mathrm{a}}$ \\
\hline 4 ou mais & $1,11(0,94-1,31)$ & & $0,96(0,79-1,15)$ & \\
\hline Situação conjugal & & $0,487^{\mathrm{a}}$ & & $0,391^{\mathrm{a}}$ \\
\hline Com cônjuge & $0,95(0,82-1,10)$ & & $0,93(0,80-1,09)$ & \\
\hline \multicolumn{5}{|l|}{ Socioeconômicas } \\
\hline Quintis de renda familiar mensal per capita & & $<0,001^{\mathrm{b}}$ & & $0,009^{c}$ \\
\hline $2^{\circ}$ & $0,80(0,66-0,96)$ & & $0,80(0,66-0,97)$ & \\
\hline $3^{\circ}$ & $0,69(0,52-0,91)$ & & $0,73(0,56-0,96)$ & \\
\hline $4^{\circ}$ & $0,68(0,48-0,98)$ & & $0,76(0,53-1,09)$ & \\
\hline $5^{\circ}$ & $0,56(0,43-0,73)$ & & $0,63(0,47-0,83)$ & \\
\hline Escolaridade & & $0,175^{\mathrm{c}}$ & & $0,355^{\mathrm{c}}$ \\
\hline Ensino médio & $1,16(0,99-1,37)$ & & $1,13(0,95-1,33)$ & \\
\hline Ensino superior ou mais & $0,94(0,65-1,36)$ & & $0,96(0,68-1,34)$ & \\
\hline Ocupação & & $0,623^{\mathrm{a}}$ & & $0,950^{\mathrm{a}}$ \\
\hline Trabalha & $0,96(0,84-1,11)$ & & $1,00(0,85-1,16)$ & \\
\hline \multicolumn{5}{|l|}{ 2o nível ( $\mathrm{n}=874)$} \\
\hline \multicolumn{5}{|l|}{ Situação de saúde $e^{* *}$} \\
\hline Número de DCNT autorreferidas & & $0,020^{\mathrm{b}}$ & & $0,022^{\mathrm{b}}$ \\
\hline $1-2$ & $1,05(0,90-1,22)$ & & $1,11(0,92-1,33)$ & \\
\hline 3 ou mais & $1,23(1,05-1,45)$ & & $1,31(1,03-1,67)$ & \\
\hline Estado nutricional & & $0,504^{\mathrm{a}}$ & & $0,650^{\mathrm{a}}$ \\
\hline Excesso de peso & $1,06(0,90-1,24)$ & & $1,05(0,86-1,27)$ & \\
\hline Insegurança alimentar & & $<0,001^{\mathrm{a}}$ & & $<0,001^{\mathrm{a}}$ \\
\hline Sim & $2,45(2,13-2,82)$ & & $2,28(1,93-2,69)$ & \\
\hline
\end{tabular}

um estudo de base de serviços de saúde incluindo usuários da atenção básica, houve uma maior proporção de mulheres na amostra e que os resultados não podem ser generalizáveis a adultos e idosos. E ainda, a realização das entrevistas na sala de espera das unidades básicas pode ter interferido nos resultados, sub ou superestimando algumas variáveis.

Entre os pontos positivos, devemos destacar o baixo percentual de perdas e o poder estatístico apropriado para a maioria das comparações realizadas. Destacamos também o fato de ser um estudo que aporta novo conhecimento ao abordar as dificuldades existentes para ter uma alimentação saudável entre usuários da rede básica.
A prevalência do desfecho (31,1\% dos participantes), embora inferior ao observado em países da União Europeia, onde 66 a 93\% manifestaram ter dificuldades para alimentação saudável ${ }^{26}$, possivelmente em virtude dos diferentes tipos de amostra e, de aspectos culturais, geográficos e econômicos da população, vem ao encontro do padrão alimentar e de morbidade dos brasileiros $^{2,3,4}$ e merece a atenção de profissionais e de autoridades em saúde.

Entre os fatores de risco observamos, que o sexo feminino, de forma semelhante ao verificado por outros pesquisadores ${ }^{16,26,27}$, mostrou-se associado ao desfecho, o que pode ser em função de as mulheres serem mais preocupadas com a 


\begin{tabular}{|c|c|c|c|c|}
\hline Variáveis & RP (IC95) & $\mathbf{p}$ & RP (IC95) & $\mathbf{p}$ \\
\hline \multicolumn{5}{|l|}{ 3o nível $(\mathrm{n}=982)$} \\
\hline Conhecimento sobre saúde e nutrição ${ }^{* * *}$ & & $0,101^{\mathrm{a}}$ & & $0,055^{\mathrm{a}}$ \\
\hline \multicolumn{5}{|l|}{ Ouviu falar nos Dez Passos da Alimentação } \\
\hline \multicolumn{5}{|l|}{ Saudável } \\
\hline Não & $1,27(0,95-1,68)$ & & $1,32(0,99-1,74)$ & \\
\hline Recebe informações sobre saúde & & $0,785^{\mathrm{a}}$ & & $0,573^{\mathrm{a}}$ \\
\hline Não & $1,03(0,86-1,23)$ & & $0,94(0,76-1,16)$ & \\
\hline Recebe informações sobre nutrição & & $0,084^{\mathrm{a}}$ & & $0,394^{\mathrm{a}}$ \\
\hline Não & $1,15(0,98-1,34)$ & & $1,09(0,90-1,31)$ & \\
\hline Recebe orientação para alimentação saudável & & $<0,001^{\mathrm{a}}$ & & $0,001^{\mathrm{a}}$ \\
\hline Não & $0,71(0,59-0,84)$ & & $0,75(0,63-0,89)$ & \\
\hline \multicolumn{5}{|l|}{ Serviços de saúde } \\
\hline Modelo de atenção à saúde utilizado & & $0,567^{\mathrm{a}}$ & & $0,936^{\mathrm{a}}$ \\
\hline Tradicional & $0,95(0,79-1,14)$ & & $1,00(0,81-1,21)$ & \\
\hline \multicolumn{5}{|l|}{ 4º nível $(\mathrm{n}=918)$} \\
\hline \multicolumn{5}{|l|}{ Hábitos de vida ${ }^{* * * *}$} \\
\hline Hábito de ler rótulos de alimentos & & $<0,001^{\mathrm{b}}$ & & $0,010^{\mathrm{b}}$ \\
\hline Às vezes & $1,49(1,22-1,83)$ & & $1,37(1,08-1,75)$ & \\
\hline Nunca & $1,56(1,23-2,00)$ & & $1,42(1,08-1,86)$ & \\
\hline Hábito de fumar & & $0,006^{\mathrm{a}}$ & & $0,343^{a}$ \\
\hline Sim & $1,30(1,08-1,56)$ & & $1,11(0,90-1,36)$ & \\
\hline Consumo de bebida alcoólica & & $0,329^{a}$ & & $0,399^{a}$ \\
\hline Sim & $0,89(0,70-1,12)$ & & $0,91(0,73-1,14)$ & \\
\hline Prática de atividade física & & $0,052^{\mathrm{a}}$ & & $0,201^{\mathrm{a}}$ \\
\hline Não & $1,26(1,00-1,58)$ & & $1,13(0,94-1,37)$ & \\
\hline
\end{tabular}

DCNT: doenças crônicas não transmissíveis. Testes: ${ }^{\mathrm{a}}$ qui-quadrado; ${ }^{\mathrm{b}}$ tendência linear; ${ }^{\mathrm{c}}$ heterogeneidade. Categorias de referência: sexo masculino; 60 anos ou mais de idade; cor da pele branca; 1-3 pessoas no domicílio; sem cônjuge; $1^{\circ}$ quintil de renda; ensino fundamental; não trabalha; nenhuma DCNT autorreferida; eutrofia; sem insegurança alimentar; ouviu falar nos Dez Passos; recebe informações sobre saúde; recebe informações sobre nutrição; recebeu orientação para alimentação saudável; atendido pela Saúde da Família; sempre lê rótulo de alimentos; não fuma; não consome bebida alcoólica; pratica atividade física;

" 2 perdas; ${ }^{* *} 372$ perdas; ${ }^{* * *} 264$ perdas; ${ }^{* * * *} 328$ perdas.

saúde e a alimentação ${ }^{28,29}$, muitas vezes por questões ligadas à estética. Tal observação pode indicar ainda ser necessária uma maior aproximação dos homens com os serviços de saúde, pois é possível que entre eles a percepção de dificuldades para ter uma alimentação saudável seja menor em decorrência do menor conhecimento e preocupação com questões ligadas, não só à alimentação saudável, mas à saúde como um todo.

Entre os adultos a verificação de uma maior probabilidade de dificuldades para uma alimentação saudável, similarmente a outros estu$\operatorname{dos}^{26,27}$, pode ser devido a que nessa fase da vida, em geral, a preocupação com os hábitos de vida, incluindo os alimentares, é menos frequente ${ }^{28-30}$.

Os usuários com maior renda apresentaram menor probabilidade de terem dificuldades para uma alimentação saudável, achados estes esperados e semelhantes à literatura ${ }^{16}$, pois é sabido que o padrão alimentar de uma população é influenciado pelo seu poder aquisitivo ${ }^{28,31}$.

A morbidade revelou ser um fator associado à percepção de dificuldade. Observamos que quanto maior o número de DCNT maior a percepção de dificuldades. Tendo em vista que a alimentação faz parte do tratamento não farmacológico de todas as DCNT que consideramos neste estudo, e que a amostra foi constituída de usuários da atenção básica, esse resultado sugere que os portadores de DCNT estejam sendo orientados, e que as orientações predominantemente restritivas aplicadas à maioria dos casos, esteja levando à percepção aumentada de dificuldades para ter uma alimentação saudável.

A insegurança alimentar, como era de se esperar, tendo em vista que sua aferição leva em consideração a disponibilidade de recursos financeiros para a compra de alimentos, se mostrou 
associada à ocorrência do desfecho, concordando com a associação verificada entre este e a renda dos participantes, conforme discutido acima.

O fato de receber ou não orientação sobre alimentação saudável influencia a percepção de dificuldade de tê-la, mas na direção diferente ao esperado, já que quem não recebeu orientação teve menor probabilidade de perceber dificuldade. Esse efeito observado não pode ser devido à confusão produzida pelo número de DCNT presentes porque foi ajustada para esse fator e porque, ainda que não tenhamos estudado todas as doenças crônicas possíveis, as RP das análises bruta e ajustada não são muito diferentes entre si, mostrando que se há confusão o efeito é desprezível. O motivo pode ser que quem não recebe orientação não perceberia que não está realizando uma alimentação saudável e, portanto, não notaria a dificuldade em tê-la.

Entre aqueles que às vezes ou nunca liam rótulos de alimentos, a percepção de dificuldades para alimentação saudável foi maior. Isso levanta a hipótese de que a leitura dos rótulos pode realmente ter uma função educativa e de facilitação do planejamento necessário para se ter uma alimentação saudável.

Quanto aos motivos para as dificuldades prevaleceram os fatores econômicos (custo elevado), seguido dos comportamentais (força de vontade insuficiente, necessidade de abrir mão de alimentos considerados não saudáveis) e finalizando com os fatores relacionados à organização do dia a dia (falta de tempo) e à falta de informação (conhecimento insuficiente).

São poucas as pesquisas brasileiras que estudam os motivos para não se ter uma alimentação saudável entre usuários de serviços de saúde ou na população geral. A maior parte dos estudos realizados ocorreu em contextos culturais e sociais diferentes aos do Brasil, o que deve ser levado em conta quando comparamos resultados, e que podem explicar as diferenças observadas em relação ao Brasil.

Dificuldades ligadas ao custo dos alimentos foram levantadas na Romênia $(78 \%)^{17}$ e na Arábia Saudita $(60,2 \%)^{18}$, caracterizando este motivo como um problema universal, que se mantém mesmo com possibilidade de diferenças em função das características de cada país, como anteriormente mencionado. Esse importante aspecto deve ser levado em consideração nas ações de promoção da alimentação saudável. No presente estudo isso é reforçado pela associação verificada entre os fatores renda e insegurança alimentar com o desfecho. Em nível individual é possível que tais dificuldades possam ser minimizadas, orientando-se, por exemplo, o consumo de alimentos da época. No âmbito coletivo, a solução de tais dificuldades abrange políticas para uma melhor distribuição de renda e controle de preço dos alimentos.

Entretanto, outro aspecto a ser considerado é a possibilidade de que exista, entre os usuários da atenção básica, uma ideia equivocada do que é uma alimentação saudável, tendo em vista que a maioria não ouviu falar dos Dez Passos da Alimentação Saudável, os quais compreendem, por exemplo, menor consumo de refrigerantes, sucos e guloseimas em geral, de sal e aumento da ingestão de água, passos estes que não dependem da renda e não têm relação com o custo dos alimentos.

Com relação aos motivos comportamentais, a força de vontade insuficiente foi importante para a população da Arábia Saudita $(80,3 \%)^{18}$ e, embora com prevalência bastante inferior, foi o terceiro motivo mais apontado por europeus $(18 \%)^{26}$. Ao mesmo tempo, abrir mão de alimentos considerados não saudáveis foi um dos principais motivos de dificuldades mencionados por romenos $(78 \%)^{17}$ e o segundo mais frequente entre europeus $(23 \%)^{26}$.

As elevadas prevalências de motivos comportamentais referidos como dificuldades para alimentação saudável apontam para a interferência de razões outras, que não o preço, nas escolhas alimentares, o que vem ao encontro dos pressupostos do modelo transteórico na abordagem do comportamento alimentar ${ }^{14}$ e reforçam a conhecida complexidade do processo de mudança ${ }^{15}$. Esse aspecto desafia os profissionais de saúde a modificarem a sua prática, em geral, padronizada e predominantemente informativa, normativa e proibitiva, no sentido de verdadeiramente estimular a mudança de hábitos, o que, por sua vez, exige também que o sistema de saúde ofereça adequadas condições de recursos para que isso se concretize.

A falta de tempo destacou-se no estudo realizado na Arábia Saudita $(67,6 \%)^{18}$, na Romênia $(34 \%)^{17}$ e na Espanha $(29,7 \%)^{16}$. Os resultados do nosso estudo apontam que, embora não seja o principal motivo, a falta de tempo também é uma barreira para a alimentação saudável entre os usuários da atenção básica. Esse achado era esperado considerando o comportamento observado na sociedade moderna, onde muitas vezes não sobra tempo para cuidar da saúde e da alimentação.

O conhecimento insuficiente apresentou prevalências mais elevadas na Arábia Saudita 
$(46,3 \%)^{18}$, na Romênia $(30 \%)^{17}$, ao passo que na Europa foi de $7 \%{ }^{26}$. Embora o conhecimento insuficiente tenha sido o tipo de dificuldade menos prevalente em nosso estudo, a maior parte dos entrevistados referiu não ter acesso a informações sobre nutrição, mas, por outro lado, mencionou acesso a informações sobre saúde. Isso sugere que a maioria dos usuários da atenção básica sabe quais são os principais elementos de uma alimentação saudável, muito provavelmente em função da atuação dos próprios profissionais de saúde. Entretanto, segundo a literatura, o padrão alimentar da população brasileira demonstra que esse conhecimento não está se traduzindo em escolhas alimentares mais adequadas ${ }^{9}$.

Por fim, gostaríamos de enfatizar que a caracterização de que uma parte dos usuários da atenção básica percebeu dificuldades em ter uma alimentação saudável, deixa claro que a política de informação nutricional deve ser melhorada, em particular entre usuários homens, adultos e com menor renda, o que inclui a implementação de novas ações que possam ser facilmente executados nos serviços de saúde visando a mudanças efetivas.

\section{Colaboradores}

IL Lindemann participou da concepção e da execução do projeto, da análise e interpretação dos dados e da redação do artigo; RR Oliveira participou da execução do projeto, da análise e interpretação dos dados e da redação do artigo; RA Mendoza-Sassi participou da concepção do projeto, da análise e interpretação dos dados, da revisão crítica do conteúdo intelectual e da aprovação final da versão a ser publicada. 


\section{Referências}

1. World Health Organization. Global strategy on diet, physical activity and health, 2004. [acessado 2010 jul 27]. Disponível em: http://apps.who.int/gb/ebwha/ pdf_files/WHA57/A57_R17-en.pdf

2. Santos RP, Horta PM, Souza CS, Santos CA, Oliveira HBS, Almeida LMR, Santos LC. Aconselhamento sobre alimentação e atividade física: prática e adesão de usuários da atenção primária. Rev Gaucha Enferm 2012; 33(4):14-21.

3. Jaime PC, Silva ACF, Lima AMC, Bortolini GA. Ações de alimentação e nutrição na atenção básica: a experiência de organização no Governo Brasileiro. Rev Nutr 2011; 24(6):809-824.

4. Olinto MT, Gigante DP, Horta B, Silveira V, Oliveira I, Willet W. Major dietary patterns and cardiovascular risk factors among young Brazilian adults. Eur J Nutr 2012; 51(3):281-291.

5. Silva SM, Facchini LA, Tomasi E, Piccini R, Thumé E, Silveira DS, Siqueira FV, Dilélio AS, Nunes BP, Saes MO. Recebimento de orientação sobre consumo de sal, açúcar e gorduras em adultos: um estudo de base nacional. Rev Bras Epidemiol 2013; 16(4):995-1004.

6. American Diabetes Association. Standards of Medical Care in Diabetes 2014. Diabetes Care 2014; 37(Supl. 1):14-80.

7. World Health Organization (WHO). Diet, nutrition and the prevention of chronic diseases: report of a Joint WHO/FAO Expert Consultation. Geneva: WHO; 2002.

8. Monteiro CA, Mondini L, Costa RBL. Mudanças na composição e adequação nutricional da dieta familiar nas áreas metropolitanas do Brasil (1988-1996). Rev Saude Publica 2000; 34(3):251-258.

9. Instituto Brasileiro de Geografia e Estatística. Pesquisa de Orçamentos Familiares (POF) 2008-2009. Análise do consumo alimentar pessoal no Brasil. Rio de Janeiro: IBGE; 2011. [acessado 2011 out 12]. Disponível em: http://www.ibge.gov.br/home/estatistica/populacao/ condicaodevida/pof/2008_2009_encaa/default.shtm

10. Brasil. Ministério da Saúde (MS). Secretaria de Atenção à Saúde. Departamento de Atenção Básica. Política Nacional de Alimentação e Nutrição. Brasília: MS; 2012. (Série B. Textos Básicos de Saúde).

11. Brasil. Ministério da Saúde (MS). Secretaria de Atenção à Saúde. Departamento de Atenção Básica. Coordenação Geral da Política de Alimentação e Nutrição. Guia alimentar para a população brasileira: promovendo a alimentação saudável. Brasília: MS; 2006. (Série A. Normas e Manuais Técnicos).

12. Santos KMO, Barros Filho AA. Fontes de informação sobre nutrição e saúde utilizadas por estudantes de uma universidade privada de São Paulo. Rev Nutr 2002; 15(2):201-210.

13. Rangel-S ML, Lamego G, Gomes ALC. Alimentação saudável: acesso à informação via mapas de navegação na internet. Physis Rev Saude Coletiva 2012; 22(3):919939.

14. Toral N, Slater B. Abordagem do modelo transteórico no comportamento alimentar. Cien Saude Colet 2007; 12(16):1641-1650.

15. Prochaska JO, DiClemente CC, Norcross JC. In Search of How People Change. Applications to Addictive Behaviors. Am Psychol 1992; 47(9):1102-1114.
16. López-Azpiazu I, Martínez-González MA, Kearney J, Gibney M, Martínez JA. Perceived barriers of, and benefits to, healthy eating reported by a Spanish national sample. Public Health Nutr 1999; 2(2):209-215.

17. Petrovici DA, Ritson C. Factors influencing consumer dietary health preventative behaviours. BMC Public Health 2006; 6:222.

18. Alquaiz AM, Tayel SA. Barriers to a healthy lifestyle among patients attending primary care clinics at a university hospital in Riyadh. Ann Saudi Med 2009; 29(1):30-35.

19. Serour M, Alqhenaei H, Al-Saqabi S, Mustafa AR Ben-Nakhi A. Cultural factors and patients' adherence to lifestyle measures. Br J Gen Pract 2007; 57(537):291295.

20. Brasil. Ministério da Saúde. Secretaria de Atenção à Saúde. Departamento de Atenção Básica. Coordenação Geral de Alimentação e Nutrição. Política Nacional de Alimentação e Nutrição. Publicações. Protocolos do Sistema de Vigilância Alimentar e Nutricional, 2008. [acessado 2012 jan 23]. Disponível em: http://189.28.128.100/nutricao/docs/geral/protocolo_sisvan.pdf

21. Brasil. Ministério da Saúde (MS). Secretaria de Vigilância em Saúde. Secretaria de Atenção à Saúde. Política Nacional de Promoção da Saúde. 3a ed. Brasília: MS; 2010. (Série B. Textos Básicos de Saúde. Série Pactos pela Saúde 2006, 7).

22. Instituto Brasileiro de Geografia e Estatística. [acessado 2014 maio 10]. Disponível em: http://www.cidades.ibge.gov.br/xtras/temas.php?lang $=$ \&codmun $=$ 431440\&idtema $=16 \&$ search $=$ rio-grande-do-sul pelotas|sintese-das-informacoes

23. Bickel G, Nord M, Price C, Hamilton W, Cook J. Measuring food security in the United States. Guide to measuring household food security. Revised 2000. Alexandria: United States Department of Agriculture; 2000.

24. Brasil. Ministério da Saúde. Secretaria de Atenção à Saúde. Departamento de Atenção Básica. Coordenação Geral de Alimentação e Nutrição. Alimentação saudável. Ciclo da vida. Desenvolvimento de habilidades pessoais. Alimentação saudável para todos: siga os dez passos. [acessado 2012 jan 19]. Disponível em: http://189.28.128.100/nutricao/docs/geral/10passosAdultos.pdf

25. Victora CG, Huttly SR, Fuchs SC, Olinto MTA. The role of conceptual frameworks in epidemiological analysis: a hierarchical approach. Int J Epidemiol 1997; 26(1):224-227.

26. Kearney JM, McElhone S. Perceived barriers in trying to eat healthier - results of a pan-EU consumer attitudinal survey. Br J Nutr 1999; 81(Supl. 2):133-137.

27. Lappalainen R, Saba A, Holm L, Mykkanen H, Gibney MJ. Difficulties in trying to eat healthier: descriptive analysis of perceived barriers for healthy eating. European Journal of Clinical Nutrition 1997; 51(Supl. 2):3640.

28. Beydoun MA, Wang Y. Do nutrition knowledge and beliefs modify the association of socio-economic factors and diet quality among US adults? Prev Med 2008; 46(2):145-153. 
29. Vinholes DB, Assunção MCF, Neutzling MB. Frequency of healthy eating habits measured by the 10 Steps to Healthy Eating score proposed by the Ministry of Health: Pelotas, Rio Grande do Sul State, Brazil. Cad Saude Publica 2009; 25(4):791-799.

30. Zart VB, Aerts D, Rosa C, Béria JU, Raymann BW, Gigante LP, Figueiredo A. Cuidados alimentares e fatores associados em Canoas, RS, Brasil. Epidemiol Serviços Saude 2010; 19(2):143-154.

31. Viebig RF, Pastor-Valero M, Scazufca M, Menezes PR. Consumo de frutas e hortaliças por idosos de baixa renda na cidade de São Paulo. Rev Saude Publica 2009; 43(5):806-813.

Artigo apresentado em 20/10/2014

Aprovado em 19/06/2015

Versão final apresentada em 21/06/2015 